\title{
A combination of alpha lipoic acid and calcium hydroxycitrate is efficient against mouse cancer models: Preliminary results
}

\author{
LAURENT SCHWARTZ ${ }^{1}$, MOHAMMAD ABOLHASSANI ${ }^{2}$, ADELINE GUAIS $^{3}$, EDWARD SANDERS ${ }^{3}$, \\ JEAN-MARC STEYAERT ${ }^{4}$, FREDERIC CAMPION $^{3}$ and MAURICE ISRAËL ${ }^{5}$ \\ ${ }^{1}$ AP-HP, Hôpital Pitié-Salpétrière, Service de radiothérapie, Paris, France; \\ ${ }^{2}$ Pasteur Institute of Iran, Tehran, Iran; ${ }^{3}$ Biorébus, Paris; ${ }^{4}$ Ecole Polytechnique, \\ Laboratoire d'informatique, Palaiseau; ${ }^{5} 2$ av Aristide Briand, Bures sur Yvette, France
}

Received December 1, 2009; Accepted December 21, 2009

DOI: $10.3892 /$ or_00000778

\begin{abstract}
The impact of metabolic dysregulation on tumor development has long been established. We have targeted two enzymes that are altered during carcinogenesis: pyruvate dehydrogenase (PDH), which is down-regulated, and ATP citrate lyase, which is overexpressed in cancer cells. Alpha lipoic acid is a cofactor of PDH, while hydroxycitrate is a known inhibitor of ATP citrate lyase. Our hypothesis is that a combination of these drugs may have antitumoral potential. The efficacy of these molecules was screened in vitro by treatment of different human cancer and murine cell lines. Lipoic acid reduced the cell number by $10-50 \%$ depending on concentrations $(0.1-10 \mu \mathrm{M})$ and cell types. Calcium hydroxycitrate reduced the cell number by $5-60 \%$ at different concentrations $(10-500 \mu \mathrm{M})$. When hydroxycitrate and lipoic acid were used together, there was a major cytotoxic effect: complete cell death was seen following $8 \mu \mathrm{M}$ lipoic acid and $300 \mu \mathrm{M}$ hydroxycitrate treatment for $72 \mathrm{~h}$. The combination of alpha lipoic acid and hydroxycitrate was administered to healthy mice, at doses currently utilized for other indications than cancer; no demonstrable toxicity was observed. The combination was used to treat mouse syngenic cancer models: MBT-2 bladder transitional cell carcinoma, B16-F10 melanoma and LL/2 Lewis lung carcinoma. The efficacy of this combination appears similar to conventional chemotherapy (cisplatin or 5-fluorouracil) as it resulted in significant tumor growth retardation and enhanced survival. This preliminary study suggests that this combination of drugs is efficient against cancer cell proliferation both in vitro and in vivo. A clinical trial is warranted.
\end{abstract}

Correspondence to: Dr Laurent Schwartz, Service de Radiothérapie Hôpital Pitié-Salpétrière, bd. de l'Hôpital, 75013 Paris, France

E-mail: laurent.schwartz@polytechnique.edu

Key words: metabolic therapy, pyruvate dehydrogenase, ATP citrate lyase

\section{Introduction}

Since the seminal work of Otto Warburg (1), it is known that cancer cells are characterized by an increased uptake of glucose and an elevated secretion of lactic acid (frequently called the Warburg effect). Although first reported by Warburg more than 80 years ago, this finding was considered to have little value with respect to the treatment of cancer. This has changed in recent years, and there are a number of excellent recent reviews on the subject (2-4). One of the major reasons for the renewal of interest in the Warburg effect is the now requent use of positron emission tomography (PET) as a diagnostic tool for cancer (5). Using a radiolabeled analog of glucose, ${ }^{18} \mathrm{~F}$-2-fluoro-2-deoxy-D-glucose, PET scan examination is able to confirm the abnormal increased uptake of large quantities of glucose by cancer cells, a consequence of increased glucose utilization.

It has often been assumed that the Warburg effect in cancer is a consequence of the hypoxic environment of tumor cells, which may or may not be the case, and that therefore a cancer must adopt an energy inefficient metabolic process in order to survive. However, Warburg clearly showed that many cancer cells actually metabolise glucose directly to lactic acid, failing to utilize the tricarboxylic acid (TCA or Krebs) cycle, even under normoxic conditions. As a consequence, this altered metabolism is often referred to as aerobic glycolysis (2). Warburg was convinced that the cause of this metabolic change in cancer was due to damage to the mitochondria that prevented oxidative phosphorylation (TCA cycle). However, this has clearly been shown not to be the case, in that many types of cancers do indeed utilize oxidative phosphorylation in the metabolism of glucose $(6,7)$. Although the actual cause of the Warburg effect is still under intense debate, there is general agreement that this metabolic alteration provides a proliferative advantage to the cancer cell $(2,8)$. There is considerable evidence that this proliferative advantage results from a redirection of glucose metabolism that allows the cancer cell to maximize the synthesis of key biomolecules, such as nucleic acids and lipids. There is also a prevailing opinion that the redirection of glucose metabolism is under the control of a variety of oncogenic proteins, such as Akt (9), c-Myc (10), and Ras (11). Just as there is now considerable 
interest in the role of the glycolytic phenotype as a key target for the development of effective drugs for cancer, the inflammatory response has also been recognized as a potential target $(12,13)$. Our laboratory has in the past few years investigated the role of hyperosmolarity as a cause of inflammation and its potential link to cancer (14-16) and most recently showed that the inhalation of carbon dioxide, a product of cellular respiration, was able to induce inflammation of the lung (17). As a consequence, focusing on metabolic changes involving glucose metabolism that characterize cancer cells appeared to us to be a logical extension of our work in the area of inflammation.

Numerous changes to the enzymes involved in aerobic glycolysis in cancer cells as compared to normal cells have been documented. Some of these changes, however, are particularly important. Four instances are: 1) Cells that exhibit the glycolytic phenotype utilize the hexokinase II (occasionally hexokinase I) isoform for the metabolism of glucose to glucose-6-phosphate, since this isoform can bind to mitochondria allowing rapid transfer of ATP across the mitochondrial membrane and also inhibits apoptosis (18). 2) Pyruvate kinase (PK), which is found almost exclusively in the M2 isoform (M2-PK) in cancer cells characterized by aerobic glycolysis. The M2 isoform, present in the embryo but essentially absent in most developed cells, exists as an active tetramer and an inactive dimer. When M2-PK is inactive, the phosphometabolites upstream of phophoenolpyruvate, the substrate for PK, accumulate thereby increasing the metabolic flux through the pentose phosphate pathway and providing the cancer cells with increased levels of ribose supplying the building blocks for nucleic acids required for the increased cellular proliferation of cancer cells (19). 3) Pyruvate dehydrogenase $(\mathrm{PDH})$, the enzyme that transforms pyruvate to acetyl-CoA providing the link between glycolysis and the Krebs cycle, is invariably inactivated in cancer cells that utilize aerobic glycolysis. Not only does this inactivation prevent complete oxidation of pyruvate through the Krebs cycle, but there is also evidence that inactivation of pyruvate dehydrogenase can stabilize HIF- $1 \alpha$ under normoxic conditions, which, in turn, can upregulate the activity of virtually every enzyme in the glycolytic pathway $(20,21) .4)$ ATP citrate lyase, which functions to degrade citrate, exported to the cytoplasm in a truncated Krebs cycle that is often associated with cancer cells exhibiting a glycolytic phenotype, to acetyl-CoA. This pathway allows cancer cells to increase lipid synthesis, which, as is the case with nucleic acid synthesis, is required to support cellular proliferation of cancer cells (22). Our hypothesis was that a synergistic targeting on several metabolic cancer cells specific alterations would be an efficient strategy to inhibit cancer growth.

In this study, we decided to target both pyruvate dehydrogenase and ATP citrate lyase, because of the results obtained by Bonnet et al (23) and Hatzivassiliou et al (22). Bonnet et al reactivated $\mathrm{PDH}$ with dichloroacetate, which inactivates pyruvate dehydrogenase kinase, the inhibitor of $\mathrm{PDH}$, $(23,24)$ and managed to prevent and inhibit tumor growth in established cancer models. Furthermore, Koukourakis et al have reported either a significant reduction of $\mathrm{PDH}$ protein levels or a significant increase of PDH kinase 1 levels (PDHK1) in human resected lung cancer tissues $(20,25)$. We chose to target restoration of PDH activity through the use of alpha lipoic acid (ALA), also a known inhibitor of PDHK1 (26).

ATP citrate lyase has been reported to be enhanced in breast, bladder, and lung carcinoma (27-29). In cancerous cells, it converts the citrate produced in the first step of the Krebs cycle into acetyl-CoA following export of citrate into the cytoplasm, which can in turn be converted into lipids required for cellular proliferation. Knock-down of ATP citrate lyase with siRNA or inhibition with the known inhibitor SB204990 has already been shown to inhibit growth of A549 human cancer cell (22). We chose to inhibit ATP citrate lyase by using calcium hydroxycitrate (HCA), a known inhibitor (30-32). The mechanistic details with respect to the biological pathways involving both lipoic acid and hydroxycitrate will be outlined in the Discussion section.

The purpose of this study was to evaluate the effect in vivo of alpha lipoic acid and/or hydroxycitrate on the growth of tumors obtained from various types of cancer cells transplanted into mice. Moreover, the results are compared to a reference treatment, either cisplatin or 5-fluorouracil.

\section{Materials and methods}

Cell cultures. The human colon (HT-29 and SW-480) and bladder (T24 and RT-112) cancer cell lines (respective ATCC numbers HTB-38, HTB-4, and CCL-228 and ECACC number 85061106) and murine bladder cancer cell line (MBT-2, kind gift of INSERM group 03-33, Créteil, France) were cultivated in Dulbecco's minimal essential medium (DMEM, Gibco) supplemented with $10 \%$ decomplemented fetal bovine serum (FBS, Eurobio, Les Ulis, France) and $1 \%$ non-essential amino acids, in a humid atmosphere with $5 \% \mathrm{CO}_{2}$ at $37^{\circ} \mathrm{C}$. To assess cell proliferation, tumor cells were seeded in 6-well plates at 20,000 cells/well in $2 \mathrm{ml}$ of culture medium for $48 \mathrm{~h}$. The culture medium was then replaced by fresh medium containing the compounds to be tested or a vehicle control. The number of living cells in the treatment group was then counted using trypan blue and normalized to the number of cells in the vehicle-treated plates after $72 \mathrm{~h}$ of incubation.

Chemicals. All chemicals were purchased from Sigma. Lipoic acid is soluble in ethanol ( $0.5 \%$ final concentration) and calcium hydroxycitrate (Garcinia cambogia extract) in water. Lipoic acid and hydroxycitrate were either diluted in culture medium for in vitro experiments or in saline solution $(9 \mathrm{~g} / \mathrm{l})$ for in vivo administration.

The doses of lipoic acid and calcium hydroxycitrate administered in vivo were chosen to be below the NOAELs (no observed adverse effect levels), based on doses previously administered to humans, and calculated by using the human/ mouse conversion table provided by the FDA (33).

Animals. All experiments were carried out in accordance with the institution recommendations based on guidelines for the care and use of laboratory animals. $\mathrm{C} 3 \mathrm{H}$ (6 weeks old) and C57BL/6 mice (8 weeks old) were obtained from the Pasteur Institute of Iran. Mice were euthanized when animals were cachexic, suffering, or when tumor volume reached $2000 \mathrm{~mm}^{3}$. 
Toxicological study. For toxicological experiments, C3H strain mice ( $\mathrm{n}=8 /$ group) were administered lipoic acid $(10 \mathrm{mg} / \mathrm{kg})$ and calcium hydroxycitrate $(250 \mathrm{mg} / \mathrm{kg})$ i.p. twice a day for 21 days. The vital function of the mice including survival, weight, and behavior were measured twice a week during the treatment and for two weeks thereafter.

Tumor models. For the bladder cancer model, we implanted $10^{6}$ MBT-2 cells subcutaneously in the back of $\mathrm{C} 3 \mathrm{H}$ mice $(\mathrm{n}=11 /$ group). The tumor developed for 9 days (mean tumor volume: $20 \mathrm{~mm}^{3}$ ). Mice were randomized for either treatment by calcium hydroxycitrate and lipoic acid (respectively 250 and $10 \mathrm{mg} / \mathrm{kg}$, twice a day) or vehicle (ethanol in saline solution $9 \mathrm{~g} / \mathrm{l}$, twice a day) or 5-fluorouracil (5-FU), as positive control $(10 \mathrm{mg} / \mathrm{kg}$, once a day during the first 4 days) $(34,35)$. Treatment with combination was administered intraperitoneally from day 9 to 54 and, to assess if treatment recovery was able to inhibit tumor growth, again continuously from day 74 until death.

For the melanoma cancer model, we implanted $10^{6}$ B16-F10 cells subcutaneously in the back of C57BL/6 mice $(n=10 /$ group). The tumor developed for 18 days (mean tumor volume: $124 \mathrm{~mm}^{3}$ ). Mice were randomized for either treatment by calcium hydroxycitrate and lipoic acid (respectively 250 and $10 \mathrm{mg} / \mathrm{kg}$, twice a day) or vehicle (ethanol in saline $9 \mathrm{~g} / \mathrm{l}$, twice a day) or cisplatin (CIS), as positive control $(1 \mathrm{mg} / \mathrm{kg}$ once a day, every two days) (36). Treatment was continuously administered intraperitoneally from day 18 until death.

For the lung carcinoma cancer model, we implanted $10^{6}$ LL/2 cells subcutaneously in the back of C57BL/6 mice $(n=10 /$ group). The tumor developed for 5 days (mean tumor volume: $323 \mathrm{~mm}^{3}$ ). Mice were randomized for either treatment by calcium hydroxycitrate and lipoic acid (respectively 250 and $10 \mathrm{mg} / \mathrm{kg}$, twice a day) or vehicle (ethanol in saline solution $9 \mathrm{~g} / \mathrm{l}$, twice a day) or cisplatin, as positive control ( $1 \mathrm{mg} / \mathrm{kg}$ once a day, every two days) (37). The combined lipoic acid and hydroxycitrate treatment was administered intraperitoneally from day 6 to day 27 .

Tumor response. We analyzed in vivo tumor growth and measured response to treatment using several parameters. Animal weight and tumor size were measured twice a week. Tumour volume in $\mathrm{mm}^{3}$ was calculated from the measurement of two perpendicular diameters using a calliper according to the formula $\mathrm{Lx} \mathrm{S} 2 / 2$, where $\mathrm{L}$ and $\mathrm{S}$ are the largest and smallest diameters in $\mathrm{mm}$, respectively (38). Time to threshold, the time required for a tumor to reach a given volume, was determined for each group. Mouse survival until time of death was also recorded. The mean tumor volume was expressed as mean relative tumor volume (mRTV), $\mathrm{mRTV}=$ $\mathrm{mV} / \mathrm{mV}_{0}$, where $\mathrm{mV}$ is the mean tumor volume on a given day and $\mathrm{mV}_{0}$ is the initial mean tumor volume on day 0 of treatment.

Data analysis. Results are reported as means and standard deviation (SD). Normality of the data was evaluated with the Kolmogorov-Smirnov test. Culture data were analyzed with univariate ANOVA after log transformation. Tumor volume data were linearized by cube root transformation. Comparison of the effect of treatments on tumor growth was performed by repeated measures general linear modeling (GLM) with weight at implantation as a covariate. Time-to-failure (i.e. when tumor volume reaches $2000 \mathrm{~mm}^{3}$ ) was analyzed with univariate ANOVA. Significance level was set at 0.05 and was maintained at this level using the Sidak procedure to adjust for the multiplicity of tests of significance. All statistical analyses were performed with SPSS v. 16.0 (SPSS Inc., Chicago, IL, USA). Drawing on published tumor growth data from xenograft studies, we estimated the sample size necessary to detect a $25 \%$ difference with the passive control group, with an overall alpha of 0.05 . The calculation by G*Power (39) indicated that repeated measures GLM with within-between interaction of 3 or 4 groups of 10 subjects or 5 groups of 11 subjects would have a power of 0.99 .

\section{Results}

We first exposed the human T24 bladder cancer cells and the human HT29 colorectal cancer cells to lipoic acid (ALA) or to calcium hydroxycitrate (HCA) in culture (Fig. 1). All doses of ALA or HCA significantly reduced cellular growth compared to control with the exception of T-24 treated with $0.1 \mu \mathrm{M}$ ALA and HT-29 treated with $10 \mu \mathrm{M}$ HCA. There was a decrease in growth of over one-third in the plates treated with either $10 \mu \mathrm{M}$ lipoic acid or with $500 \mu \mathrm{M}$ calcium hydroxycitrate compared to plates treated with only the vehicle.

We then combined the two drugs (ALA+HCA) using doses of each drug that had significantly reduced cellular growth compared to control. We obtained an additive effect: the use of $4 \mu \mathrm{M}$ lipoic acid plus $200 \mu \mathrm{M}$ calcium hydroxycitrate resulted in only 27\% (T24) and 8.5\% (HT-29) living cells after $72 \mathrm{~h}$ of incubation. An increase in lipoic acid and calcium hydroxycitrate concentrations ( $8 \mu \mathrm{M}$ lipoic acid plus $300 \mu \mathrm{M}$ calcium hydroxycitrate) resulted in a striking cytotoxic effect with no surviving cells (T24 and HT-29). Similar results were obtained with other human (RT-112 and SW480) or murine (MBT-2) cancer cells (data not shown).

The next step was to confirm that the treatment was innocuous. Healthy mice were treated for three weeks twice daily by the coadministration of the hydroxycitrate $(250 \mathrm{mg} / \mathrm{kg} /$ twice a day) and lipoic acid (10 mg/kg/twice a day) combination. No effects on weight, behavior, and survival of the animals were observed through two weeks after the end of the treatment (data not shown).

We decided to analyse the effect of hydroxycitrate and lipoic acid combination on tumor development. For technical reasons, human cell xenografts in mice were not available, so we used syngeneic models. From our point of view, the choice of the tumor origin was not an important element because there seems to be a general agreement at this time that the Warburg effect is present in the large majority of cancer whatever the site of origin and whatever the carcinogen responsible $(2,8,40,41)$. However, we selected cell lines for which the literature described evidence of the Warburg effect.

Several studies described a relationship between the cMyc pathway and the glycolytic pathway (reviewed in ref. 42). Among the available cell lines the MBT-2 bladder carcinoma overexpressed the cMyc gene. Furthermore, the down- 


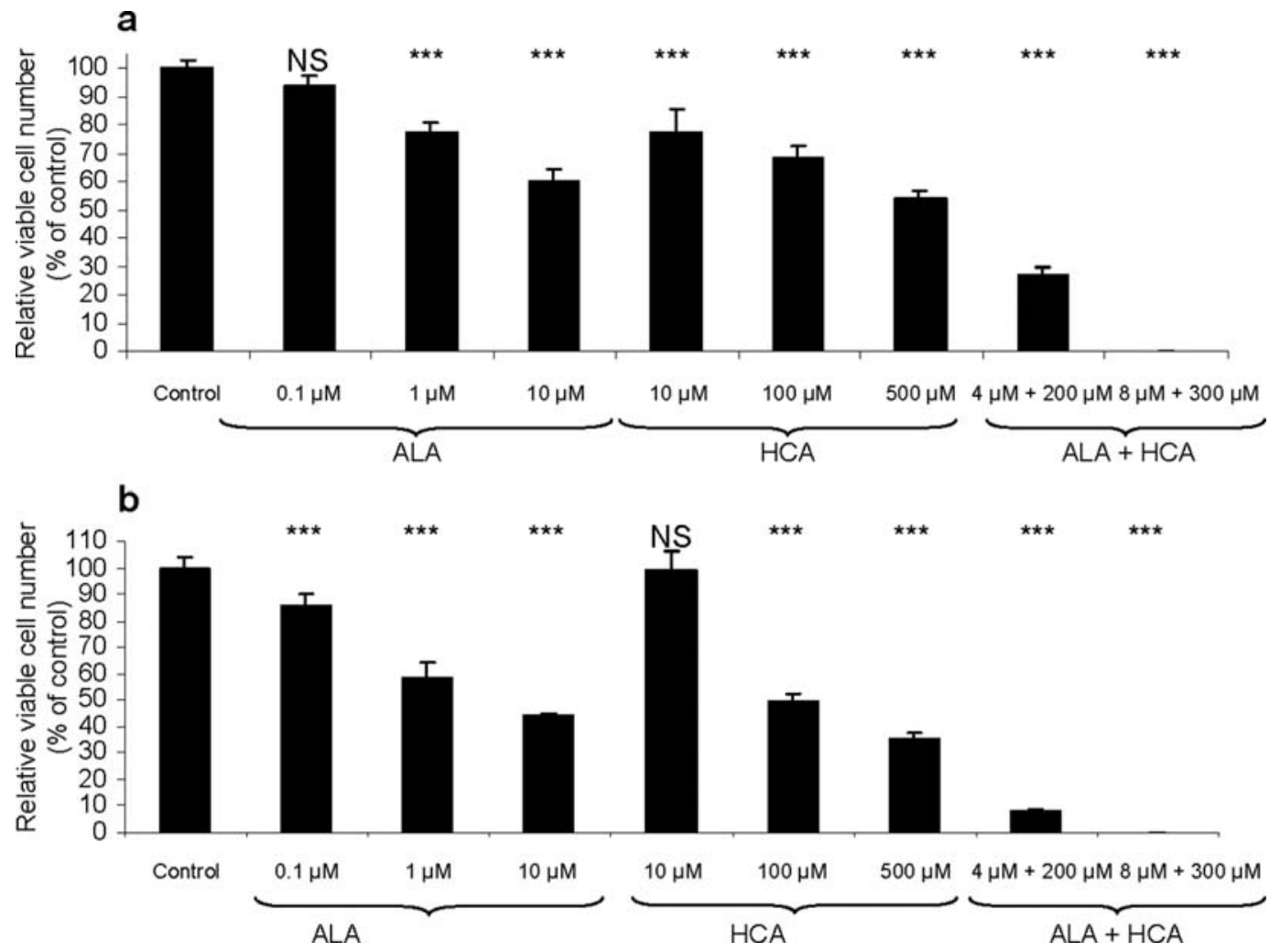

Figure 1. Major in vitro cytotoxicity of the lipoic acid and calcium hydroxycitrate combination. T24 (a) and HT-29 (b) cancer cell lines were cultured for three days in the presence of various concentrations of lipoic acid (ALA) or/and calcium hydroxycitrate (HCA). Cellular viability was assessed by trypan blue coloration followed by direct cell counting. For each concentration, the graphs present the viable cell number normalized to control. For each experiment, there are three wells per condition and each well is counted twice. The graphs present the average value ( \pm standard deviation) of one representative experiment; results were reproduced three times independently. With the exception of T-24 treated with $0.1 \mu \mathrm{M}$ ALA alone (NS, P=0.612) and HT-29 treated with $10 \mu \mathrm{M}$ HCA alone (NS, $\mathrm{P}=1.0$ ), all doses of ALA or HCA, either alone or in combination, significantly reduced cellular growth compared to control $\left({ }^{* * *} \mathrm{P}<0.001\right)$.

regulation of the cMyc protein and lactate dehydrogenase A transcription was described in MBT-2 tumor models efficiently treated with imidazolquinoline (43).

Therefore, we first injected murine MBT-2 cancer cells into $\mathrm{C} 3 \mathrm{H}$ mice (Fig. 2). Analysis of transformed tumor volume data over 35 days using general linear models with HuynhFeldt correction showed that the time-by-treatment interaction of the five groups (saline, ethanol, 5-FU, ALA, and ALA+HCA) differed significantly $\left(\mathrm{F}_{8.4,103}=6.88, \mathrm{P}<0.001\right)$ (Fig. 2a). Post-hoc analysis with Sidak correction shows that the differences between 5-FU and ALA+HCA (-11.7, P=0.7, $95 \%$ CI -33.6 to 10.2) were not significant. However over 50 days there was a significant time-by-treatment interaction between 5-FU and ALA+HCA $\left(\mathrm{F}_{2.4,46.0}=39.62, \mathrm{P}<0.001\right)$.

Comparison of 5-FU and ALA+HCA over 50 days indicated a significant time-by-interaction difference in the treatment $\left(\mathrm{F}_{11.8,90.8}=20.4 .62, \mathrm{P}<0.001\right)$. At day 50, animals treated with 5-FU had a larger tumor size than those treated with ALA+HCA $\left(30.9 \mathrm{~mm}^{3}, \mathrm{P}=0.017,95 \%\right.$ CI 5.6 to 74.2$)$ (Fig. 2b).

We observed a slowing of tumor growth from day 19 in the 5-FU (positive control) and lipoic acid/calcium hydroxycitrate treated mice, whereas the negative controls (saline and ethanol) rapidly developed a $2000 \mathrm{~mm}^{3}$ tumor (40 days after inoculation). Furthermore, while 5-FU treated tumors reached $2000 \mathrm{~mm}^{3}$ in 55 days, the lipoic acid/calcium hydroxycitrate group did not reach the $2000 \mathrm{~mm}^{3}$ threshold before 86 days (Fig. 2b).

Tumor growth was significantly delayed in the combination group as compared to vehicle or 5-FU controls. The difference in tumor growth rate between treatments and positive control appeared by day 25: 5-FU $(\beta=-38.9, \mathrm{P}=0.003)$, ALA $(\beta=-25.1, P=0.036)$, and ALA + HCA $(\beta=-24.2$, $\mathrm{P}=0.043)$. At day 40 , growth rates of $5-\mathrm{FU}(B=-300.5)$ and ALA+HCA $(B=-333.4)$ treated tumors were significantly lower (both $\mathrm{P}<0.001)$ compared to control. The growth rate of 5-FU became significantly higher than ALA+HCA at day $45(\beta=118.9, \mathrm{P}<0.001)$ and continued to increase at day 60 $(\beta=486.9, P<0.001)$. It is also interesting to note that the lipoic acid/hydroxycitrate treatment increased the lifespan of the mice by a factor of two as compared to controls. Time to failure (i.e. when tumor volume reaches $2000 \mathrm{~mm}^{3}$ ) in the MBT-2 model was 39.5 (2.4) for controls, 43.0 (1.7) for ethanol, 46.1 (5.1) for ALA, 51.9 (2.3) for 5-FU, and 89.1 (5.1) for ALA+HCA. The group treated with 5-FU had longer time to failure than negative controls $(12.4, \mathrm{P}<0.001$, 95\% CI 7.9 to 16.9$)$ and $\mathrm{ALA}(5.8, \mathrm{P}=0.004 ; 95 \% \mathrm{CI} 1.3$ to 10.3) but lower than ALA+HCA $(-37.0, \mathrm{P}<0.001 ; 95 \% \mathrm{CI}$ 41.6 to -32.5$)$.

In a second in vivo cancer model, we chose to target B16F10 melanoma because it has been described that this model overexpressed the pyruvate kinase and phophoglycerate kinase 

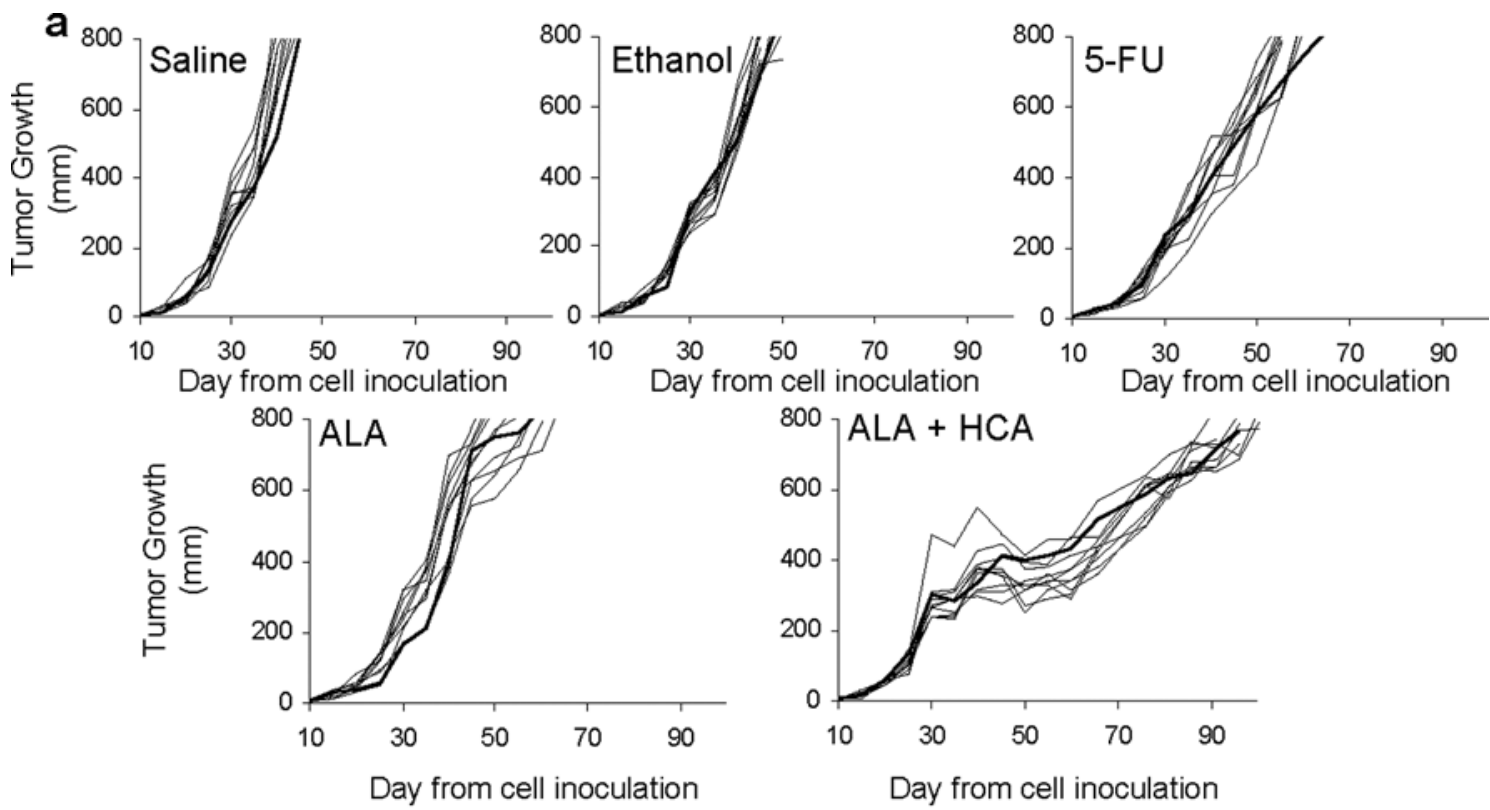

b

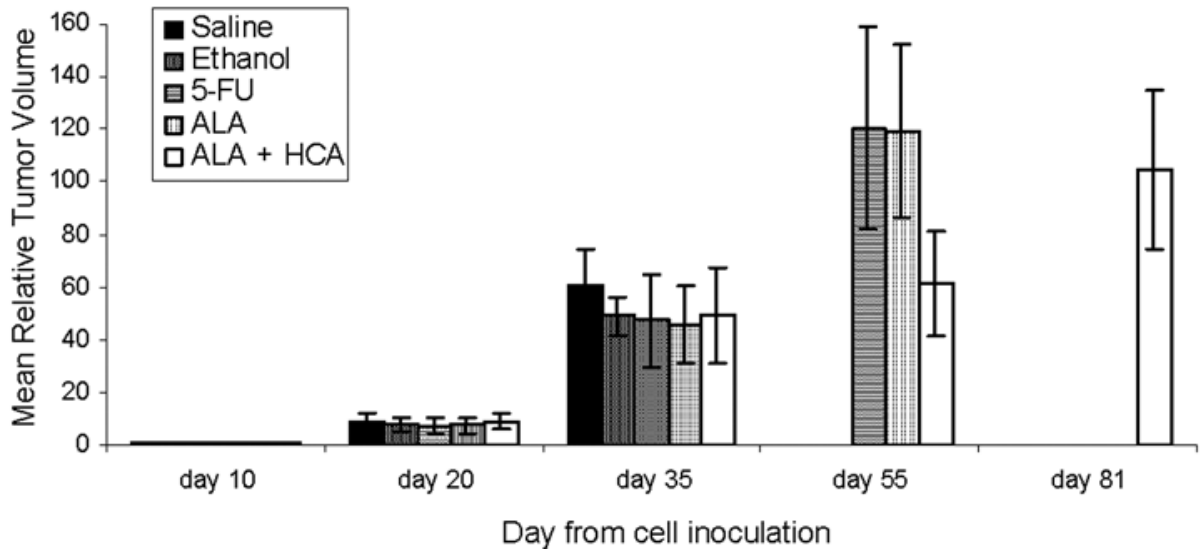

Figure 2. In vivo antitumor activity of the lipoic acid and calcium hydroxycitrate combination on the MBT-2 cancer model. Mice (n=11/group) were implanted with MBT-2 bladder cancer cells and treatments were administered intraperitoneally every 12 h from day 9 after implantation until day 54 and again continuously from day 74 until sacrifice. (a) Tumor growth (see Materials and methods for details) curves for individual mouse in each treatment group. (b) Mean relative tumor volume (with SD) at different time points after cell inoculation. Lipoic acid/calcium hydroxycitrate combination (ALA+HCA) caused tumor growth delay as compared to controls (saline, ethanol), 5-fluorouracil (5-FU) or lipoic acid (ALA) treated groups.

genes during its development (44). We implanted B16-F10 melanoma cells in C57BL/6 mice and analysed the effect of lipoic acid and hydroxycitrate combination (Fig. 3). We switched to cisplatin, as active control, because in this cancer model (and also in the third), 5-FU does not appear to be effective. The time-by-treatment interaction was significantly different between groups (control, ethanol, cisplatin and ALA+HCA) over 42 days $\left(\mathrm{F}_{6.4,74.6}=9.7, \mathrm{P}<0.001\right)$. Post-hoc analysis with Sidak correction showed that the difference between cisplatin and ALA+HCA is not significant $\left(10.8 \mathrm{~mm}^{3}\right.$ $\mathrm{P}=0.993 ; 95 \% \mathrm{CI}-40.5$ to 62.1 ). At day 37 , in comparison to negative control, growth rate was significantly lower in ALA+HCA $(\beta=-68.8, P<0.034)$ but not in cisplatin $(\beta=-34.4$, $\mathrm{P}=0.273$ ) (Fig. 3a). At day 57, growth rate of ALA+HCA and cisplatin were significantly lower than control $(B=-748.0$ and $\beta=-419.5$ respectively, both $\mathrm{P}<0.001)$. Consistent with the previous model, we observed a slowing of tumor growth from day 30 in cisplatin and lipoic acid/calcium hydroxycitrate treated mice, whereas the negative controls (saline and ethanol) rapidly developed a $2000 \mathrm{~mm}^{3}$ tumor. Tumor growth was significantly delayed in the combination group as compared to vehicle or cisplatin. Time to failure in the B16-F10 model was respectively 47.4 days (SD1.6) in saline, 48.6 (1.7) in ethanol, 51.4 (1.6) in cisplatin, and 62.1 (1.6) in ALA+HCA groups. No difference in time to failure was found between control and cisplatin $(4.0, \mathrm{P}=0.403,95 \% \mathrm{CI}-2.2$ to 10.2$)$ while ALA+HCA reached the threshold later than cisplatin (10.7, $\mathrm{P}<0.001,95 \% \mathrm{CI}-4.4$ to 17.0 ) (Fig. 3b).

As a third in vivo cancer model, we used LL/2 lung carcinoma because it was demonstrated that the viability of these cells was strongly hampered following a reduction of activity of the glycolytic enzymes caused by clotrimazole treatment $(45,46)$. Thus, LL/2 (Lewis) carcinoma cells were implanted in C57BL/6 mice (Fig. 4). A significant difference in time-by-treatment interaction $\left(\mathrm{F}_{2.6,33.6}=19.3, \mathrm{P}<0.001\right)$ was found between groups (saline, cisplatin, and ALA+HCA) 

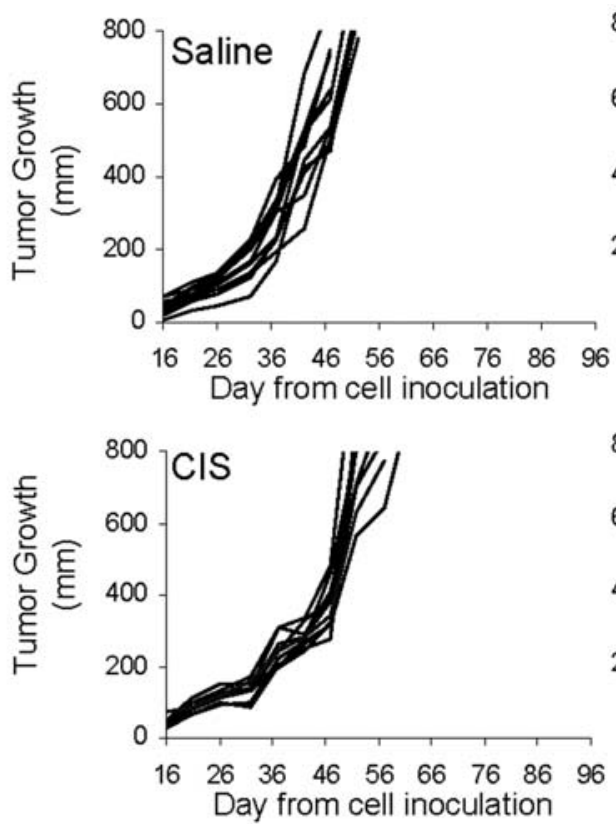
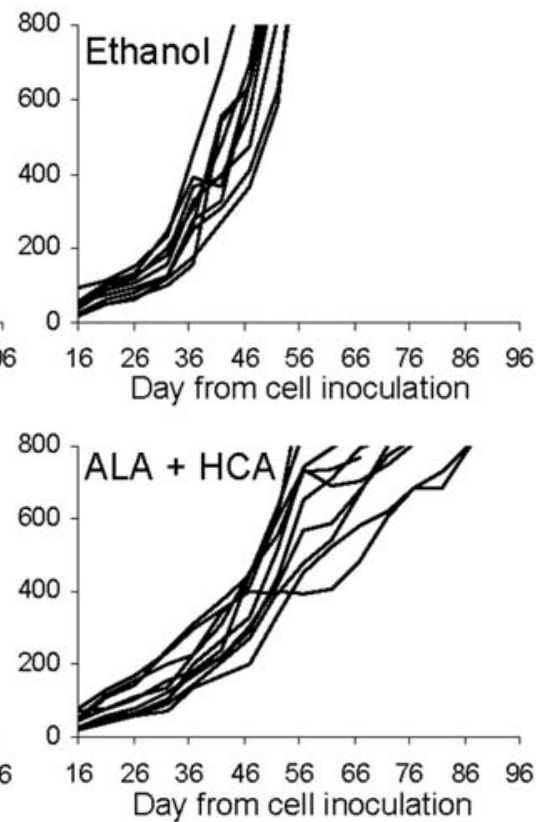

b

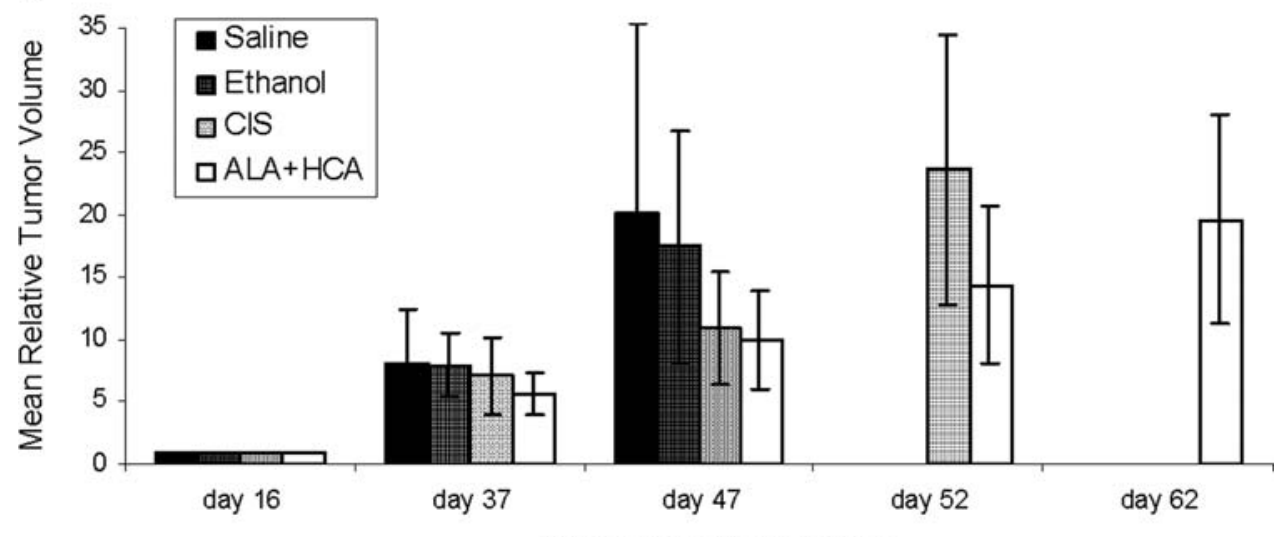

Day from cell inoculation

Figure 3. In vivo antitumor activity of the lipoic acid and calcium hydroxycitrate combination on the B16-F10 cancer model. Mice (n=10/group) were implanted with B16-F10 melanoma cells and treatments were administered intraperitoneally every $12 \mathrm{~h}$ from day 18 after implantation until sacrifice. (a) Tumor growth curves for individual mice in each treatment group. (b) Mean relative tumor volume (with SD) at different time points after cell inoculation. Lipoic acid/calcium hydroxycitrate combination (ALA+HCA) caused tumor growth delay as compared to control (saline, ethanol), or cisplatin (CIS) treated groups.

over 15 days. The tumor volume was found to be larger in cisplatin than in ALA+HCA in post-hoc analysis with Sidak correction $\left(38.1 \mathrm{~mm}^{3}, \mathrm{P}=0.05,95 \% \mathrm{CI}-0.3\right.$ to 76.4$)$. Consistent with the previous models, we observed a slowing of tumor growth from day 12 in the mice treated either with cisplatin or with the lipoic acid/calcium hydroxycitrate combination (Fig. 4a). The difference in tumor growth rate between treatments and control appeared by day 15 : cisplatin $(\beta=-89.1$, $\mathrm{P}=0.005)$ and ALA+HCA $(\beta=-174.9, \mathrm{P}<0.001)$. In the LL-2 model, time to failure in control was $17.1(1.0)$, in cisplatin 21.9 (1.0), and $23.2(1.0)$ in ALA+HCA. Cisplatin reached the threshold later than negative control $(4.8, \mathrm{P}=0.015,95 \% \mathrm{CI}$ 0.7 to 8.9) while the difference with ALA+HCA was not significant (-1.4, $\mathrm{P}=0.928 ; 95 \% \mathrm{CI}-5.5$ to 2.7 ) (Fig. 4b).

An analysis with repeated measures GLM of log transformed body weights among the treatment groups in the three models revealed a significant difference: $\mathrm{F}_{3.24}=1.81, \mathrm{P}=0.013$ for $\mathrm{B} 16-\mathrm{F} 10, \mathrm{~F}_{3.16}=8.40, \mathrm{P}<0.001$ for $\mathrm{LL} 2$, and $\mathrm{F}_{4.32}=16.18$, $\mathrm{P}<0.001$ for MBT-2, respectively. The body weight of animals treated with ALA+HCA was systematically higher but the difference with animals treated with cisplatin or 5-FU which increased with time did not reach significance.

\section{Discussion}

The main finding is that a combination of alpha lipoic acid and hydroxycitrate strongly reduces tumor growth. Time to failure of animals is extended compared to those treated with cisplatin or 5-FU. There are differences in terms of apparent efficacy inbetween the three cancer models: the time to failure is reached later in the MBT-2 model than in the B16F10, which is also reached later than in the LL/2 model. This can be related to the tumor development kinetics of each model: the MBT-2 bladder cancer evolve quite slowly as 


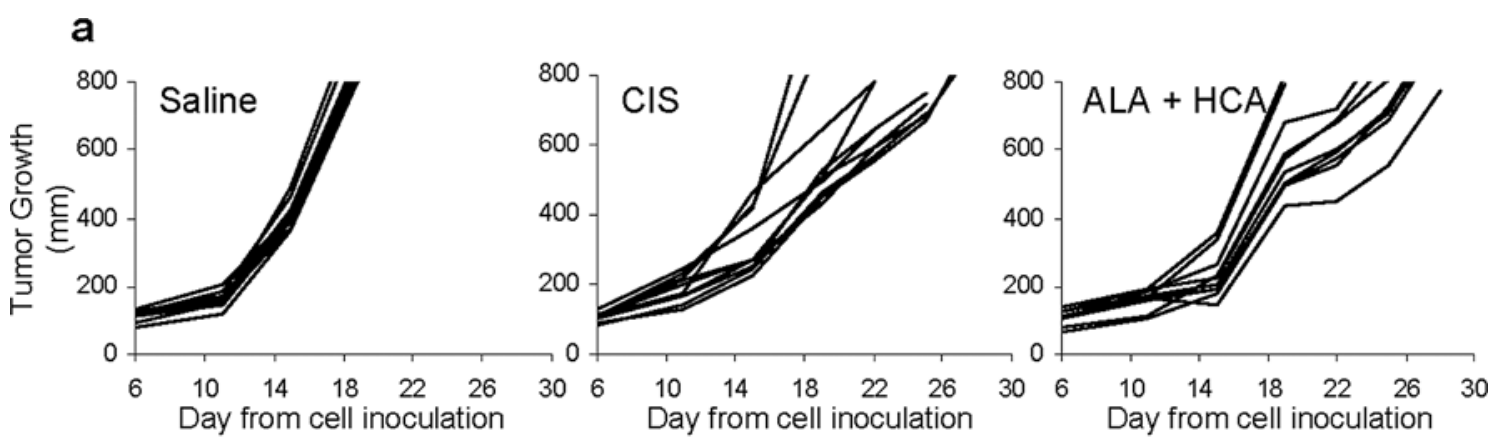

b

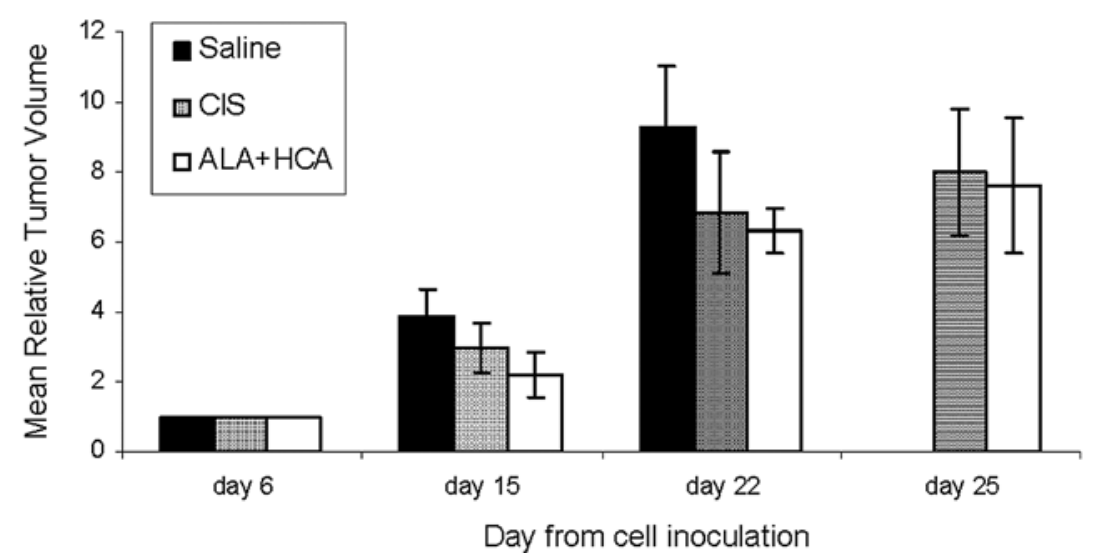

Figure 4. In vivo antitumor activity of the lipoic acid and calcium hydroxycitrate combination on the LL/2 cancer model. Mice (n=10/group) were implanted with LL/2 lung carcinoma cells, and treatments were administered intraperitoneally every 12 h from day 6 to day 27 . (a) Tumor growth curves for individual mice in each treatment group. (b) Mean relative tumor volume (with SD) at different time points after cell inoculation. Lipoic acid/calcium hydroxycitrate combination (ALA+HCA) caused tumor growth delay as compared to control (saline) or cisplatin (CIS) treated groups.

compared to the two others. Moreover, the tumor volumes were not the same at the beginning of the drug administration; and, an early treatment will be more effective than a later one. Thus, we can not conclude from these preliminary data on the efficacy of the combination of drugs in relation to the different tumor types.

Cancer cells have a clearly altered metabolism. They display an increased uptake of glucose and, among other alterations, an increase in lactic acid production, and decrease in pyruvate kinase and pyruvate dehydrogenase activities, and an increase in ATP citrate lyase activity. In this study, we targeted the last two enzymes.

Pyruvate dehydrogenase complex (PDC) is a large multienzyme complex composed of several copies of pyruvate dehydrogenase (E1), dihydrolipoyl transacetylase (E2), and dihydrolipoyl dehydrogenase (E3). Pyruvate dehydrogenase kinase (PDHK) is an integral part of the PDC, with two or three PDHK dimers per complex. Inactivation of the E1 component of the PDC complex occurs when PDHK phosphorylates three serine residues on E1 (ser264, ser271 and ser203) (47). It has been shown that lipoic acid restores the activity of PDH through the inhibition of PDHK (26).

The fact that our results showed lipoic acid was able to inhibit tumor growth suggests that restoration of PDH activity allows pyruvate to be metabolized to acetyl-CoA, thereby reducing the amount of lactate produced by the tumor cells. There is considerable evidence that production of lactate can provide an advantage to the growth of tumor cells, perhaps through creating an acidic environment which is damaging to normal cells $(48,49)$. This is exactly the logic that was suggested by McFate et al (50), who inactivated PDHK in a human squamous cell head and neck cancer cell line through the use of a specific shRNA thus apparently restoring the PDH activity, reverting the Warburg metabolic phenotype, and inhibiting growth of tumors obtained by xenografting these cells into nude mice.

Several studies suggest that lipoic acid can indeed inhibit the growth of cancer cells that show a glycolytic phenotype. A publication by Van de Mark et al (51) reported that lipoic acid initiated apoptosis in the FaDu and Jurkat human cell lines as well is in a Ki-v-Ras transformed Balb/c-3T3 murine mesenchymal cell line where apoptosis was not induced in normal cells. Similarly, when HT-29 human colon, H460 human lung epithelial cancer cells as well as two hepatoma cell lines (murine FaO, human HepG2 and SMMC-7721 cells) and human promyelocytic HL-60 cells were exposed to lipoic acid, apoptosis was observed (52-56).

ATP citrate lyase (ACL) is a key enzyme involved in lipid biosynthesis, but only recently has it been linked to the Warburg effect. Perhaps the first study to explore the role of ACL in the production of lipids in cancer cells was published by Hatzivassiliou et al (22). These authors showed that inhibition of ACL, using both siRNA and the specific inhibitor SB-204990, was able to inhibit cancer cell growth both in vitro and in vivo, using xenografts in nude mice, and that in general levels of ACL are significantly elevated in these tumor cell 
lines. A rather large assortment of different cell lines was investigated. Cancer cells displaying high rates of glucose metabolism are more severely affected by the two treatments, whereas those displaying a low rate of aerobic glycolysis are largely unaffected, indicating that the ability of ACL inhibition to suppress tumor growth correlates with the glycolytic phenotype of the tumor. It is important to note that ACL is the enzyme that converts citrate to acetyl-CoA, which is then used as the building block for lipid synthesis.

To the best of our knowledge, no experiment demonstrating hydroxycitrate efficacy against cancer has ever been published, although one in vitro study demonstrated that calcium hydroxycitrate capacity to interfer with the pyruvate metabolism in tumorigenic cells (57). This study examined pyruvate metabolism in four cell lines, two tumorigenic cell lines (H.Ep2 and ESH TRI-1) a suppressed cell line (ESH p6), and MRC5, an untransformed diploid fibroblast cell line from a fetal lung. Treatment of these cells with hydroxycitrate resulted in a marked decrease (4-6-fold) of pyruvate consumption in the two tumorigenic cell lines but about a two-fold increase in the MRC5 cells. However, the percentage of pyruvate that was oxidized to $\mathrm{CO}_{2}$ was increased nearly two-fold. In addition, a marked decrease in lactate production was also noted following hydroxycitrate treatment. This is consistent with the recent results of Wellen et al (58) demonstrating that one gene that appeared to be down-regulated through the inhibition of histone acetylation that occurred following the inhibition of ACL was lactate dehydrogenase A. Therefore, inhibition of ACL may not simply deprive tumor cells of lipids required for cell proliferation, it may also result in down-regulation of lactate dehydrogenase, decreasing aerobic glycolysis and allowing pyruvate to be imported into the mitochondria where it can be metablized through the Krebs cycle.

Therefore, it would appear that the approach utilized in this study may indeed result in a synergistic attack on the metabolic pathways used by these cancer cells. Lipoic acid inhibits the activity of PDHK, thereby allowing pyruvate that is imported into the mitochondria to be converted to mitochondrial acetyl-CoA by $\mathrm{PDH}$, thus allowing the Krebs cycle to proceed, while hydroxycitrate inhibits ACL, which reduces the lipid available for cell proliferation and decreases the activity of lactate dehydrogenase.

Our results suggest that targeting cellular metabolism may be an alternative to conventional cytotoxic chemotherapy. Neither hydroxycitrate nor lipoic acid are known to target DNA or to be cytotoxic to normal human cells $(59,60)$. No demonstrable toxicity due to the long-term combination of these two drugs could be observed during this study.

Lipoic acid and calcium hydroxycitrate, when administrated alone, have at best a cytostatic effect on tumor cells (Fig. 1). The rate of response is slow, clearly different from conventional chemotherapy. When the drugs are combined, however, there is a major cytotoxic effect similar to results obtained with conventional cytotoxic chemotherapy. In vitro, cancer cells are killed within hours when exposed to cytotoxic drugs (61).

In the three animal models studied, the kinetics of response to the combined metabolic treatment is similar to the positive controls (5-FU or cisplatin). Using either conventional chemotherapy or metabolic therapy, it takes at least one week to obtain a decrease in the rate of tumor growth. Metabolic therapy seems effective in delaying tumor growth in the models studied, although there are differences in the individual responses. It should be noted that the choice of dose for this first animal study was empirical. We did not demonstrate any toxicity at doses as close as possible to the plasma level reached when humans were treated either by food supplements or in case of lipoic acid when treated for neuropathy.

Both lipoic acid and calcium hydroxycitrate are well known to the clinician and are available over the counter. Since the early 1990s, lipoic acid has been widely used as a dietary supplement, typically at doses in the range of 100-200 mg/day. It has been approved as a treatment for diabetic neuropathy in Germany. The dose is usually $600 \mathrm{mg}$ per day, but higher doses have been prescribed (62). Experimental and clinical studies have indicated the potential usefulness of lipoic acid treatment as a therapeutic agent for the prevention of various pathologies including diabetes, atherosclerosis, ischemia-perfusion injury, degenerative processes in neurons, joint diseases, radiation injury, heavy metal poisoning and HIV activation (54). Furthermore, lipoic acid has been used in clinical trials as an antioxidant to fight cancer-related cachexia and oxidative stress syndrome $(63,64)$, and a case-report showed that in association with selenium and low-dose naltrexone, lipoic acid was efficacious in the long-term survival of a patient diagnosed with poorly differentiated adenocarcinoma of the pancreas (65). The therapeutic potential of lipoic acid as well as mechanisms of action has recently been reviewed (66). Hydroxycitrate, extracted from Garcinia cambogia fruit, is used as a weight loss agent because it presumably limits lipogenesis and reduces food intake, although the results obtained on human body weight regulation are controversial (67). In humans, it is usually administered at $1500 \mathrm{mg} /$ day, but $3 \mathrm{~g} /$ day has already been tested (68).

One strength of this study is the full randomization with both passive and active controls. In addition statistical tests were corrected for the fact that multiple comparisons were made. However, we acknowledge that the lack of evaluation of enzyme activity is a limitation as it made it impossible to establish and measure the effect of the experimental treatment on the targeted enzymatic pathways.

Cancer is characterized by a large number of metabolic disturbances which are linked to cancer progression, as highlighted by two recent publications $(69,70)$. The present study shows that targeting just one of these disturbances is not particularly effective, but targeting two has a striking effect. It is tempting to assume that targeting a third metabolic alteration will lead to an even more effective response. In conclusion, this study demonstrates the efficacy of targeting metabolic pathways of cancer cells to control their proliferation and may provide incentive for further investigation to clarify the mechanisms at work. It is a first step towards the possibility of developing a metabolic approach to cancer therapy.

\section{Acknowledgements}

We acknowledge the help of Philippe Chaumet-Riffaud. This work was funded by Biorébus. 


\section{References}

1. Warburg O: On The origin of cancer cells. Science 123: 309-314, 1956.

2. Vander Heiden MG, Cantley LC and Thompson CG: Understanding the Warburg effect: the metabolic requirements of cell proliferation. Science 324: 1029-1033, 2009.

3. Kroemer G and Pouyssegur J: Tumor cell metabolism: cancer's Achilles' heel. Cancer Cell 13: 472-482, 2008.

4. Gillies RJ and Gatenby RA: Adaptive landscapes and emergent phenotypes: why do cancers have high glycolysis? J Bioenerg Biomembr 39: 251-257, 2007

5. Gambhir SS, Czernin J, Schwimmer J, Silverman DHS, Coleman RE and Phelphs ME: A tabulated summary of the FDG PET literature. J Nucl Med 42: S1-S93, 2001.

6. Robey RB and Hay N: Is Akt the 'Warburg kinase'? Semin Cancer Biol 10: 25-31, 2009.

7. Moreno-Sanchez R, Rodriguez-Enriquez S, Marin-Hernandez A and Saavedra E: Energy metabolism in tumor cells. FEBS J 274: 1393-1418, 2007.

8. Gatenby RA and Gillies RJ: Why do cancers have high aerobic glycolysis? Nat Rev Cancer 4: 891-899, 2004.

9. Elstrom RL, Bauer DE, Buzzai M, Karnauskas R, Harris MR, Plas DR, Zhuang H, Cinalli RM, Alavi A, Rudin CM and Thompson CB: Akt stimulates aerobic glycolysis in cancer cells. Cancer Res 64: 3892-3899, 2004.

10. Kim J, Gao P, Liu YC, Semenza GL and Dang CV: Hypoxia inducible factor 1 and dysregulated c-Myc cooperatively induce vascular endothelial growth factor and metabolic switches hexokinase 2 and pyruvate dehydrogenase kinase 1. Mol Cell Biol 27: 7381-7393, 2007.

11. Chiaradonna F, Sacco E, Manzoni R, Giorgio M, Vanoni M and Alberghina L: Ras-dependent carbon metabolism and transformation in mouse fibroblasts. Oncogene 25: 5391-5404, 2006.

12. Balkwill $\mathrm{F}$ and Mantovani A: Inflammation and cancer: back to Virchow? Lancet 357: 539-545, 2001.

13. Schwartz L, Balosso J, Baillet F, Brun B, Amman JP and Sasco AJ: Cancer: the role of extracellular disease. Med Hypotheses 58: 340-346, 2002.

14. Abolhassani M, Wertz X, Pooya M, Chaumet-Riffaud P, Guais A and Schwartz L: Hyperosmolarity causes inflammation through the methylation of protein phosphatase $2 \mathrm{~A}$. Inflamm Res 57: 419-429, 2008

15. Schwartz L, Abolhassani M, Pooya M, Steyaert J-M, Wertz X, Israël M, Guais A and Chaumet-Riffaud P: Hyperosmotic stress contributes to mouse colonic inflammation through the methylation of protein phosphatase 2A. Am J Physiol Gastrointest Liver Physiol 295: G934-G941, 2008.

16. Schwartz L, Guais A, Pooya M and Abolhassani M: Is inflammation a consequence of extracellular hyperosmolarity? J Inflamm 6: 21, 2009

17. Abolhassani M, Guais A, Chaumet-Riffaud P, Sasco AJ and Schwartz L: Carbon dioxide inhalation causes pulmonary inflammation. Am J Physiol Lung Cell Mol Physiol 296: L657-L665, 2009.

18. Pederson PL: Warburg, me and hexokinase 2: multiple discoveries of key molecular events underlying one of cancer's most common phenotypes, the 'Warburg effect', i.e., elevated glycolysis in the presence of oxygen. J Bioenerg Biomembr 39: 211-222, 2007.

19. Mazurek S, Boschek CB, Hugo F and Eigenbrodt E: Pyruvate kinase type M2 and its role in tumor growth and spreading. Semin Cancer Biol 15: 300-308, 2005.

20. Koukourakis MI, Giatromanolaki A, Sivridis E, Gatter KC and Harris AL: Tumor and Angiogenesis Research Group: Pyruvate dehydrogenase and pyruvate dehydrogenase kinase expression in non-small cell lung cancer and tumor-associated stroma. Neoplasia 7: 1-6, 2005.

21. Lu H, Forbes RA and Verma A: Hypoxia-inducible factor 1 activation by aerobic glycolysis implicates the Warburg effect in carcinogenesis. J Biol Chem 277: 23111-23115, 2005.

22. Hatzivassiliou G, Zhao F, Bauer D, Andreadis C, Shaw AN, Dhanak D, Hingorani SR, Tuveson DA and Thompson CB: ATP citrate lyase inhibition can suppress tumor cell growth. Cancer Cell 8: 311-321, 2005.

23. Bonnet S, Archer SL, Allalunis-Turner J, Haromy A, Beaulieu C, Thompson R, Lee CT, Lopaschuk GD, Puttagunta L, Bonnet S, Harry G, Hashimoto K, Porter CJ, Andrade MA, Thebaud B and
Michelakis ED: A mitochondria- $\mathrm{K}^{+}$channel axis is suppressed in cancer and its normalization promotes apoptosis and inhibits cancer growth. Cancer Cell 11: 37-51, 2007.

24. Michelakis ED, Webster L and Mackey JR: Dichloroacetate (DCA) as a potential metabolic-targeting therapy for cancer. $\mathrm{Br}$ J Cancer 99: 989-994, 2008.

25. Koukourakis MI, Giatromanolaki A, Bougioukas G and Sivridis E: Lung cancer: a comparative study of metabolism related protein expression in cancer cells and tumor associated stroma. Cancer Biol Ther 6: 1476-1479, 2007.

26. Korotchkina LG, Sidhu S and Patel MS: R-Lipoic acid inhibits mammalian pyruvate dehydrogenase kinase. Free Radic Res 38: 1083-1092, 2004.

27. Szutowicz A, Kwiatkowski J and Angielski S: Lipogenetic and glycolytic enzyme activities in carcinoma and nonmalignant diseases of the human breast. Br J Cancer 39: 681-687, 1979.

28. Turyn J, Schlichtholz B, Dettlaff-Pokora A, Presler M, Goyke E, Matuszewski M, Kmiec Z, Krajka K and Swierczynski J: Increased, activity of glycerol 3-phosphate dehydrogenase and other lipogenic enzymes in human bladder cancer. Horm Metab Res 35: 565-569, 2003.

29. Migita T, Narita T, Nomura K, Miyagi E, Inazuka F, Matsuura M, Ushijima M, Mashima T, Seimiya H, Satoh Y, Okumura S, Nakagawa K and Ishikawa Y: ATP citrate lyase: activation and therapeutic implications in non-small cell lung cancer. Cancer Res 68: 8547-8554, 2008.

30. Berkhout TA, Havekes LM, Pearce NG and Groot PHE: The effect of (-)-hydroxycitrate on the activity of the low-densitylipoportein receptor and 3-hydroxy-3-methylglutaryl.CoA reductase levels in the human hepatoma cell line Hep G2. Biochem J 272: 181-186, 1990.

31. Senner A and Malaisse WJ: Hexose metabolism in pancreatic islets. Effect of (-)-hydroxycitrate upon fatty acid synthesis and insulin release in glucose-stimulated islets. Biochimie 73: 1287-1290, 1991

32. Lim K, Ryu S, Suh H, Ishihara K and Fushiki T: (-)-Hydroxycitrate ingestion and endurance exercise performance. J Nutr Sci Vitaminol 51: 1-7, 2005.

33. Food and Drug Administration, Center for Drug Evaluation and Research (CDER): Estimating the Maximum Safe Starting Dose in Initial Clinical Trials for Therapeutics in Adult Healthy Volunteers, Pharmacology and Toxicology, July 2005.

34. Ikemoto S, Sugimura K, Wada S, Yasumoto K, Yamamoto K and Kishimoto T: Augmentation of antitumor activity of 5'-deoxy5 -fluorouridine by thymosin fraction 5 in mouse bladder cancer cells in vitro and in vivo. Cancer Lett 145: 121-126, 1999.

35. Nishikawa T, Kohjimoto Y, Nishihata M, Ebisuno S and Hara I: Synergistic antitumor effects of fleroxacin with 5-fluorouracil in vitro and in vivo for bladder cancer cell lines. Urology 74: 1370-1376, 2009.

36. Ghosh S and Maity P: Augmented antitumor effects of combination therapy with VEGF antibody and cisplatin on murine B16F10 melanoma cells. Int Immunopharmacol 7: 1598-1608, 2007.

37. Li G, Tian L, Hou JM, Ding ZY, He QM, Feng P, Wen YJ, Xiao F, Yao B, Zhang R, Peng F, Jiang Y, Luo F, Zhao X, Zhang L, Zhou Q and Wei YQ: Improved therapeutic effectiveness by combining recombinant CXC chemokine ligand 10 with Cisplatin in solid tumors. Clin Cancer Res 11: 4217-4224, 2005.

38. Bissery MC and Chabot GG: History and new development of screening and evaluation methods of anticancer drugs used in vivo and in vitro. Bull Cancer 78: 587-602, 1991.

39. Faul F, Erdfelder E, Lang AG and Buchner A: G*Power 3: A flexible statistical power analysis program for the social, behavioral, and biomedical sciences. Behav Res Methods 39: 175-191, 2007

40. Hsu PP and Sabatini DM: Cancer cell metabolism: Warburg and beyond. Cell 134: 703-707, 2008.

41. Young CD and Anderson SM: Sugar and fat - that's where it's at: metabolic changes in tumors. Breast Cancer Res 10: 202, 2008.

42. Dang CF, Kim J, Gao P and Yustein J: The interplay between MYC and HIF in cancer. Nat Rev Cancer 8: 51-56, 2008.

43. Liu H, Schwartz MJ, Hwang DH and Scherr DS: Tumour growth inhibition by an imidazoquinoline is associated with c-Myc down-regulation in urothelial cell carcinoma. BJU Int 101: 894-901, 2008. 
44. Culp WD, Neal R, Massey R, Egevad L, Pisa P and Garland D: Proteomic analysis of tumor establishment and growth in the B16-F10 mouse melanoma model. J Proteome Res 5: 1332-1343, 2006.

45. Penso J and Beitner R: Clotrimazole decreases glycolysis and the viability of lung carcinoma and colon adenocarcinoma cells. Eur J Pharmacol 451: 227-235, 2002.

46. Penso J and Beitner R: Detachment of glycolytic enzymes from cytoskeleton of Lewis lung carcinoma and colon adenocarcinoma cells induced by clotrimazole and its correlation to cell viability and morphology. Mol Genet Metab 76: 181-188, 2002.

47. Tuganova A, Klyuaeva A and Popov K: Recognition of the inner lipoyl-bearing domain of dihydrolipyl transacetylase and of the blood glucose-lowering compound AZD7545 by pyruvate dehydrogenase kinase 2. Biochemistry 46: 8592-8602, 2007.

48. Gatenby RA, Gawlinski ET, Gmitro AF, Kaylor B and Gillies RJ Acid-mediated tumor invasion: a multidisciplinary study. Cancer Res 66: 5216-5223, 2006.

49. Israel M and Schwartz L: Cancer as a Dysmethylation Syndrome. John Libbey, Paris, 2006.

50. McFate T, Mohyelden A, Lu H, Thakar J, Henriques J, Halim ND Wu H, Schell MJ, Tsang TM, Teahan O, Zhou S, Califano JA, Jeoung NH, Harris RA and Verma A: Pyruvate dehydrogenase complex activity controls metabolic and malignant phenotype in human cancer cells. J Biol Chem 283: 22700-22708, 2008.

51. Van de Mark K, Chen JS, Steliou K, Perrine SP and Faller DV Alpha-lipoic acid induces p27Kip-dependent cell cycle arrest in non-transformed cell lines and apoptosis in tumor cell lines. J Cell Physiol 194: 325-340, 2003.

52. Wenzel U, Nickel A and Daniel H: alpha-lipoic acid induces apoptosis in human colon cancer cells by increasing mitochondrial respiration with a concomitant $\mathrm{O} 2-*_{\text {-generation. }}$ Apoptosis 10: 359-368, 2005

53. Moungjaroen J, Nimmannit U, Callery PS, Wang L, Azad N, Lipipun V, Chanvorachote P and Rojanasakul Y: Reactive oxygen species mediate caspace activation and apoptosis induced by lipoic acid in human lung epithelial cancer cells through Bcl-2 down regulation. J Pharmacol Exp Ther 319: 1062-1069, 2006.

54. Simbula G, Columbano A, Ledda-Columbano GM, Sanna L, Deidda M, Diana A and Pibiri M: Increased ROS generation and p53 activation in $\alpha$-lipoic acid-induced apoptosis of hepatoma cells. Apoptosis 12: 113-123, 2007.

55. Shi DY, Liu HL, Stern JS, Yu PZ and Liu SL: Alpha-lipoic acid induces apoptosis in hepatoma cells via the PTEN/Akt pathway. FEBS Lett 582: 1667-1671, 2008.

56. Selvakumar E and Hsieh T: Regulation of cell cycle transition and induction of apoptosis in HL-60 leukemia cells by lipoic acid: role in cancer prevention and thereapy. J Hematol Oncol 1: 4, 2008.
57. Board $\mathrm{M}$ and Newsholme E: Hydroxycitrate causes altered pyruvate metabolism by tumorigenic cells. Biochem Mol Biol Int 40: 1047-1056, 1996.

58. Wellen KE, Hatzivassiliou G, Sachdeva UM, Bui TV, Cross JR and Thompson CB: ATP-citrate lyase links cellular metabolism to histone acetylation. Science 324: 1076-1080, 2009.

59. Cremer DR, Rabeler R, Roberts A and Lynch B. Safety evaluation of alpha-lipoic acid (ALA). Regul Toxicol Pharmacol 46: 29-41, 2006.

60. Soni MG, Burdock GA, Preuss HG, Stohs SJ, Ohia SE and Bagchi D: Safety assessment of (-)-hydroxycitric acid and Super CitriMax, a novel calcium/potassium salt. Food Chem Toxicol 42: 1513-1529, 2004

61. DeVita VT, Hellman TS and Rosenberg SA: Cancer: Principles \& Practice of Oncology. 8th edition, Lippincott Williams \& Wilkins, Philadelphia, 2008.

62. Ruessmann HJ: German Society of out patient diabetes centres AND (Arbeitsgemeinschaft niedergelassener diabetologisch tätiger Arzte e.V.): Switching from pathogenetic treatment with alpha-lipoic acid to gabapentin and other analgesics in painful diabetic neuropathy: a real-world study in outpatients. J Diabetes Complications 23: 174-177, 2009.

63. Mantovani G, Macciò A, Madeddu C, Gramignano G, Serpe R, Massa E, Dessì M, Tanca FM, Sanna E, Deiana L, Panzone F, Contu $\mathrm{P}$ and Floris $\mathrm{C}$ : Randomized phase III clinical trial of five different arms of treatment for patients with cancer cachexia: interim results. Nutrition 24: 305-313, 2008.

64. Novotny L, Rauko P and Cojocel C: Alpha-lipoic acid: the potential for use in cancer therapy. Neoplasma 55: 81-86, 2008.

65. Berkson BM, Rubin DM and Berkson AJ: The long-term survival of a patient with pancreatic cancer with metastases to the liver after treatment with the intravenous alpha-lipoic acid/low-dose naltrexone protocol. Integr Cancer Ther 5: 83-89, 2006.

66. Shay KP, Moreau RF, Smith EJ, Smith AR and Hagen TM: Alpha-lipoic acid as a dietary supplement: molecular mechanisms and therapeutic potential. Biochim Biophys Acta 1790: 1149-1160, 2009.

67. Kovacs EM and Westerterp-Plantenga MS: Effects of (-)-hydroxycitrate on net fat synthesis as de novo lipogenesis. Physiol Behav 88: 371-381, 2006.

68. Downs BW, Bagchi M, Subbaraju GV, Shara MA, Preuss HG and Bagchi D: Bioefficacy of a novel calcium-potassium salt of (-)-hydroxycitric acid. Mutat Res 579: 149-162, 2005.

69. Schafer ZT, Grassian AR, Song L, Jiang Z, Gerhart-Hines Z, Irie HY, Gao S, Puigserver P and Brugge JS: Antioxidant and oncogene rescue of metabolic defects caused by loss of matrix attachment. Nature 461: 109-113, 2009.

70. Gottlieb E: Cancer: The fat and the furious. Nature 461: 44-45, 2009. 